\title{
Characteristics of peripheral immune cell subsets in patients with carotid atherosclerosis undergoing carotid endarterectomy
}

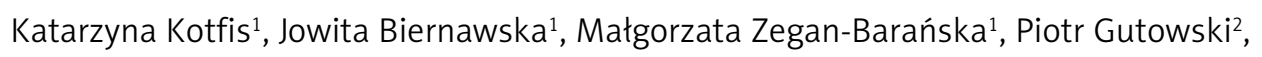
Maciej Żukowski ${ }^{1}$

'Department of Anesthesiology, Intensive Therapy and Acute Intoxications, Pomeranian Medical University, Szczecin, Poland

${ }^{2}$ Department of Vascular and General Surgery and Angiology, Pomeranian Medical University, Szczecin, Poland

Submitted: 25 July 2016

Accepted: 4 October 2018

Arch Med Sci Atheroscler Dis 2018; 3: e129-e136 DOI: https://doi.org/10.5114/amsad.2018.79537 Copyright (c) 2018 Termedia \& Banach

\section{Abstract}

Introduction: The role of circulating immune cells in the pathophysiology of cerebrovascular accidents is currently under debate. The aim of this study was to characterize peripheral immune cell subsets in patients undergoing carotid endarterectomy (CEA).

Material and methods: We conducted a prospective observational study in a group of 124 patients with significant carotid stenosis undergoing carotid endarterectomy, both symptomatic and asymptomatic. We compared the percentages of circulating immune cells: B lymphocytes, T lymphocytes, $T$ helper, cytotoxic T, CD4+/CD8+ ratio, T regulatory, monocytes and NK cells before CEA and $6 \mathrm{~h}$ after the procedure.

Results: Total lymphocyte count and cytotoxic $T$ lymphocyte count decreased $6 \mathrm{~h}$ after CEA in both subgroups. The NK cell level decrease was statistically significant only in the symptomatic subgroup (19.41 \pm 9 .30 before CEA and $16.52 \pm 9.37$ after CEA; $p=0.0044)$, but not in the asymptomatic subgroup (17.88 \pm 9.14 before CEA and $15.91 \pm 9.51$ after CEA; $p=0.0886)$. The $T$ lymphocyte level showed a statistically significant increase only in symptomatic patients (69.74 \pm 10.16 before CEA vs. $71.45 \pm 9.77$ after CEA; $p=0.0462)$, and not in the asymptomatic subgroup $(70.08 \pm 11.19$ prior to CEA and $70.21 \pm 12.35 ; p=0.9048$ ). B lymphocyte, helper $\mathrm{T}$ lymphocyte and regulatory $T$ (Treg) lymphocyte $(C D 4+/ C D 25+)$ levels showed a significant increase after CEA.

Conclusions: This is the first study to compare circulating immune cells in patients undergoing carotid endarterectomy. Only the symptomatic subgroup experienced a significant decrease in the NK cell level and an increase in the T lymphocyte count after CEA. This study enriches our understanding of immune cell kinetics during carotid endarterectomy.

Key words: leukocytes, regulatory T cells, lymphocytes, monocytes, ischemic stroke, carotid endarterectomy, immune cells, chronic cerebrovascular disease.

\section{Introduction}

Atherosclerosis is a chronic inflammatory disease of the arterial wall, including the cerebral circulation, leading to a broad spectrum of clini-

\author{
Corresponding author: \\ Katarzyna Kotfis \\ Department \\ of Anesthesiology \\ Intensive Therapy \\ and Acute Intoxications \\ Pomeranian Medical \\ University \\ Al. Powstańców Wlkp. 72 \\ 70-111 Szczecin, Poland \\ Phone: +48 602449202 \\ E-mail: katarzyna.kotfis@ \\ pum.edu.pl
}


cal symptoms, namely acute ischemic stroke (IS), transient ischemic attack (TIA), reversible ischemic neurologic deficit (RIND) and amaurosis fugax (AF) [1]. In atherogenesis the key finding is the presentation of autoantigens by dendritic cells leading to activation of anti-inflammatory and pro-inflammatory immune cells and accumulation of foam cells within the subendothelial region of the artery [2]. Treatment of patients with carotid atherosclerosis includes both non-invasive pharmacological treatment and revascularization therapy (carotid artery endarterectomy or stenting) [3].

Carotid endarterectomy (CEA) has become standard treatment, minimizing the incidence of stroke for carotid atherosclerosis, with over 150000 procedures performed each year in the USA, although the key issue is adequate patient qualification [4]. Clinical data for the role of CEA have been provided by four major multi-centre studies: the North American Symptomatic Carotid Endarterectomy Trial (NASCET), the European Carotid Surgery Trial (ECST), the Asymptomatic Carotid Atherosclerosis Trial (ACAS) and the Asymptomatic Carotid Surgery Trial (ACST) [5].

The role of circulating immune cells in the pathophysiology of cerebrovascular accidents is not fully elucidated and is currently under debate. The neuro-immunologic interactions both within the unstable atheromatous plaque and in the peripheral circulating immune cell subsets are of great importance in the pathogenesis of ischemic stroke and related symptoms. White blood cells provide an immediate response to tissue damage and serve as effectors of the immune system, each subset with a different function and differentially activated by specific stimuli. Therefore careful analysis of circulating leukocytes may become a useful tool in evaluation of the inflammatory and immune status of the patient to predict which patients are at risk of experiencing complications [6]. Immune cells most frequently implicated to be altered in patients with atherosclerosis are both lymphocyte and monocytes subpopulations $[6,7]$.

Carotid endarterectomy, serving as a major insult to the diseased carotid vessel, may alter the levels of circulating immune cells. Only a few studies have analyzed the association between circulating immune cells and advanced carotid atherosclerotic disease, with no data on the effect of CEA on the immune cell levels. The data from studies in humans are limited and concentrate on the immune composition of the atherosclerotic plaque and not on the peripheral immune cells. Although the role of innate and adaptive immune cell species is crucial in atherosclerotic plaque pathophysiology [8], almost no study has specifically investigated the regulation of these cells in patients undergoing carotid endarterectomy in patients with chronic symptomatic and asymptomatic cerebrovascular disease [9]. With atherosclerosis being a chronic inflammatory disease depending on the action of $\mathrm{T}$ lymphocytes and macrophages and their cytokines on the arteries, the appreciation of the disease dynamics in different patient groups is crucial, especially when given the fact that the spectrum of symptoms may be nonhomogeneous [10, 11].

It is a well-known fact that stroke-induced peripheral immunodepression is a common occurrence after an acute cerebrovascular accident [12]. This is a phenomenon related both to adaptive and innate immunity. It is triggered by the sympathetic activity of the vulnerable central nervous system lesions that cause catecholamine-induced apoptosis of the lymphatic organs [13-15]. Therefore, it must be appreciated that in terms of atherosclerotic immunity the immune system of symptomatic patients may respond to CEA differently than patients who were previously asymptomatic.

The role of circulating immune cells in the pathophysiology of cerebrovascular accidents has been identified as an important aspect of the problem and a possible site for interventions. The data from studies in humans are very limited and concentrate on the immune composition of the atherosclerotic plaque. The relationship between local and peripheral immune cell behavior and post-CEA outcome is being increasingly recognized and many questions about the regulation of immune cell subsets are still unanswered and have to be clarified before further translation of novel preclinical treatment strategies into clinical practice occurs $[10,11,16,17]$.

We hypothesized that the effect of a major vascular intervention, such as carotid endarterectomy, on circulating immune cells may be different in symptomatic and asymptomatic patients. The aim of this study was to characterize peripheral immune cell subsets in patients undergoing carotid endarterectomy, and compare both symptomatic and asymptomatic patients before and after the procedure to enrich the knowledge about the pathophysiology of human atherosclerosis.

\section{Material and methods}

A prospective observational study was conducted in a group of patients undergoing a planned carotid endarterectomy in the Department of Vascular Surgery and the Department of Anesthesiology, Intensive Therapy and Acute Intoxications, Pomeranian Medical University in Szczecin, Poland. The study was performed according to the Declaration of Helsinki. The approval of the institutional Ethics Committee of the Pomeranian Medical University was granted (No. BN-001/52/08) and each individual patient gave written informed consent for 
participation in this study. This article reports no individual participant's data in any form.

Patients with hemodynamically significant stenosis of the carotid artery in its extra-cerebral course undergoing a scheduled carotid endarterectomy were included in this study. The exclusion criteria were: emergency surgery; previously performed carotid endarterectomy or carotid artery stenting; concomitant inflammatory or autoimmune diseases (i.e. viral hepatitis, cirrhosis, connective tissue disorders, inflammatory bowel diseases - Crohn's disease and ulcerative colitis, multiple sclerosis, Graves-Basedow disease, Hashimoto disease); neoplasm and disorders of hematopoiesis; any surgery within the previous 6 months. All patients in the study group were on long-term prophylactic doses of aspirin (75 mg) orally.

Data regarding the patients enrolled in the study were analyzed according to their preoperative status depending on the presence or absence of neurological symptoms prior to carotid endarterectomy. The symptomatic subgroup included patients with: carotid stenosis (60-99\%) and after a cerebrovascular episode according to TOAST criteria, with clinical symptoms manifesting as either IS, RIND, TIA or AF [18]. The asymptomatic subgroup included patients with carotid stenosis $\geq 70 \%$, with no previous episodes of cerebrovascular ischemia prior to carotid endarterectomy.

All patients undergoing CEA received standard perioperative monitoring (ECG, $\mathrm{SpO}_{2}$, invasive blood pressure) and received regional anaesthesia in the form of a superficial and deep carotid plexus block using $0.5 \%$ bupivacaine $(2 \mathrm{mg} / \mathrm{kg}$ ) and $2 \%$ lidocaine $(4 \mathrm{mg} / \mathrm{kg})$. The surgical procedure involved a longitudinal incision of the carotid artery, followed by removal of the atheromatous plaque by eversion and a continuous suture of the vessel. After the procedure patients were managed according to the local protocol regarding monitoring and analgesia in the postoperative care unit.

\section{Flow cytometry}

Immune cell analysis was performed using two-color immunofluorescence flow cytometry with a monoclonal antibody (MAb) panel (Simultest - BD, San Jose, California, USA). To enable analysis a sample of whole arterial blood was collected from each patient into sterile heparinized tubes with EDTA at two time-points, i.e. before and 6 hours after carotid endarterectomy. A 30-minute incubation period followed red cell lysis and fixation in $1 \%$ formalin. All antibodies were prepared according to the directions of the manufacturer. Fluorescein isothiocyanate (FITC) and phycoerythrin (PE)-conjugated MAb for desired cell surface proteins were used: B lymphocyte - CD3-/CD19+, T lymphocyte-CD3+, Thelper lymphocyte-CD3+/
CD4+; cytotoxic T lymphocyte - CD3+/CD8+, NK cells - D3+/CD16+CD56+, CD4+ to CD8+ ratio, Treg lymphocyte - CD4+/CD25+. The analysis was performed using a flow cytometry FACSCalibur device (Becton Dickinson, USA) with CellQuest software (version 2.0, system OS2). The data were expressed as percentages of total lymphocytes \pm standard error of mean. The number of $\mathrm{CD} 4^{+} \mathrm{CD} 25^{+}$ lymphocytes was expressed as the percentage of CD4+ lymphocytes. Lymphocyte sub-populations were identified using forward-scattered light (FSC) and the side-scattered light (SSC).

\section{Statistical analysis}

The data were described using means with standard deviation (SD) or numbers and percentages. For continuous variables significant deviations from normality were verified using the Kolmogorov-Smirnov test. Difference testing between two groups was performed using Student's $t$-test for unpaired data. The Mann-Whitney test or Student's t-test was applied to paired data. The data were analyzed using STATA 11 (license no.: 30110532736) statistical software. A p-value of less than 0.05 was considered to indicate statistical significance.

\section{Results}

The study group was composed of 124 patients aged 44 to 87 years, including 46 (37\%) women and 78 (63\%) men. Both demographic and clinical data for the symptomatic and asymptomatic subgroups are depicted in Table I. The symptomatic subgroup consisted of 75 patients (24 women and 51 men), with a mean age of $68.43 \pm 9.70$ years, whereas the symptomatic subgroup consisted of 49 patients ( 22 women and 27 men), with a mean age of $68.35 \pm 8.40$ years.

Both subgroups were generally comparable, although the asymptomatic subgroup presented with more: ischemic disease (53.06\% vs. $33.33 \%$; $p=0.038)$, acute myocardial infarction $(28.57 \%$ vs. $9.33 \% ; p=0.005)$ and more $\beta$-blocker therapy (59.18\% vs. $37.84 \% ; p=0.020)$ prior to the procedure.

Data regarding the operation and anaesthesia are shown in Table II. There were no clinically or statistically significant differences between the two subgroups.

\section{Peripheral cell subset data}

The percentages of peripheral, circulating immune cells obtained using flow cytometry for both subgroups are shown in Table III. The total lymphocyte count decreased $6 \mathrm{~h}$ after CEA in both subgroups, as did the cytotoxic $T$ lymphocyte count, which was statistically significant. The NK 
Table I. Demographic and preoperative medical data for study subgroups

\begin{tabular}{|lccc|}
\hline Variables & Symptomatic $(n=75)$ & Asymptomatic $(n=49)$ & $P$-value \\
\hline Age, mean \pm SD [years] & $68.43 \pm 9.70$ & $68.35 \pm 8.40$ & 0.769 \\
\hline Male sex & $51(68.00 \%)$ & $27(55.10 \%)$ & 0.146 \\
\hline BMI, mean \pm SD $\left[\mathrm{kg} / \mathrm{m}^{2}\right]$ & $27.81 \pm 3.65$ & $27.08 \pm 4.42$ & 0.422 \\
\hline LCA \% stenosis & 65.64 & 63.37 & 0.547 \\
\hline RCA \% stenosis & 48.41 & 61.73 & 0.027 \\
\hline Ischemic stroke & $55(73.33 \%)$ & $\mathrm{NA}$ & \\
\hline TIA & $16(21.33 \%)$ & $\mathrm{NA}$ & \\
\hline RIND & 0 & $\mathrm{NA}$ & \\
\hline Amaurosis fugax & $8(10.67 \%)$ & $26(53.06 \%)$ & 0.038 \\
\hline Ischemic heart disease (yes) & $25(33.33 \%)$ & $17(34.69 \%)$ & 0.876 \\
\hline Diabetes (yes) & $25(33.33 \%)$ & $14(28.57 \%)$ & 0.005 \\
\hline Acute myocardial infarction (yes) & $7(9.33 \%)$ & $33(67.35 \%)$ & 0.947 \\
\hline Congestive heart failure (no) & $52(69.33 \%)$ & $39(79.59 \%)$ & 0.132 \\
\hline Arterial hypertension (yes) & $67(89.33 \%)$ & $25(51.02 \%)$ & 0.402 \\
\hline Dyslipidemia (yes) & $44(58.67 \%)$ & $16(32.65 \%)$ & 0.599 \\
\hline History of smoking (yes) & $21(28.00 \%)$ & $28(57.14 \%)$ & 0.087 \\
\hline ACE-I (yes) & $54(72.00 \%)$ & $29(59.18 \%)$ & 0.020 \\
\hline$\beta$-Blockers (yes) & $28(37.84 \%)$ & $28(57.14 \%)$ & 0.283 \\
\hline Statins (yes) & $50(66.67 \%)$ & & \\
\hline
\end{tabular}

$n$ - number of patients, LCA - left carotid artery, RCA - right carotid artery, BMI - body mass index, TIA - transient ischemic attack, RIND - reversible ischemic neurologic deficit, ACE-I - angiotensin converting enzyme inhibitors, NA - not applicable, SD - standard deviation, $p$ - statistical significance.

Table II. Perioperative data for study subgroups

\begin{tabular}{|lccc|}
\hline Variable & Symptomatic $(n=75)$ & Asymptomatic $(n=49)$ & $P$-value \\
\hline Procedure length, mean \pm SD [min] & $70.73 \pm 22.04$ & $67.14 \pm 16.93$ & 0.229 \\
\hline Cross-clamping time, mean \pm SD [min] & $22.44 \pm 7.86$ & $20.44 \pm 6.21$ & 0.244 \\
\hline Lidocaine use, mean \pm SD [mg] & $386.40 \pm 35.09$ & $380.63 \pm 45.68$ & 0.431 \\
\hline Bupivacaine use, mean \pm SD [mg] & $99.27 \pm 6.65$ & $98.91 \pm 8.93$ & 0.798 \\
\hline Fluids i.v., mean \pm SD [ml] & $1247.97 \pm 506.83$ & $1173.47 \pm 370.82$ & 0.379 \\
\hline
\end{tabular}

$S D$ - standard deviation, $p$ - statistical significance.

Table III. Peripheral immune cell changes before and 6 hours after CEA for the symptomatic and asymptomatic subgroups

\begin{tabular}{|c|c|c|c|c|c|c|}
\hline \multirow[t]{2}{*}{ Peripheral cells } & \multicolumn{2}{|c|}{ Symptomatic patients } & \multirow[t]{2}{*}{$P$-value } & \multicolumn{2}{|c|}{ Asymptomatic patients } & \multirow[t]{2}{*}{$P$-value } \\
\hline & $\begin{array}{l}\text { Prior to CEA } \\
\quad n=73 \\
\text { Mean } \pm \text { SD }\end{array}$ & $\begin{array}{c}6 \mathrm{~h} \text { after CEA } \\
n=48 \\
\text { Mean } \pm \text { SD }\end{array}$ & & $\begin{array}{c}\text { Prior to CEA } \\
\quad n=73 \\
\text { Mean } \pm \text { SD }\end{array}$ & $\begin{array}{c}6 \mathrm{~h} \text { after CEA } \\
n=47 \\
\text { Mean } \pm \text { SD }\end{array}$ & \\
\hline Lymphocytes (total) & $28.70 \pm 9.64$ & $19.45 \pm 8.73$ & 0.0001 & $31.71 \pm 12.25$ & $19.89 \pm 9.93$ & 0.0001 \\
\hline B lymphocytes & $10.85 \pm 5.97$ & $12.03 \pm 7.17$ & 0.0138 & $12.62 \pm 8.18$ & $14.13 \pm 8.62$ & 0.0155 \\
\hline T lymphocytes & $69.74 \pm 10.16$ & $71.45 \pm 9.77$ & 0.0462 & $70.08 \pm 11.19$ & $70.21 \pm 12.35$ & 0.9048 \\
\hline NK cells & $19.41 \pm 9.30$ & $16.52 \pm 9.37$ & 0.0044 & $17.88 \pm 9.14$ & $15.91 \pm 9.51$ & 0.0886 \\
\hline Monocytes & $5.34 \pm 1.74$ & $5.21 \pm 1.51$ & 0.5222 & $5.91 \pm 1.81$ & $5.55 \pm 1.90$ & 0.1801 \\
\hline T helper lymphocytes (CD4+) & $43.79 \pm 10.34$ & $47.39 \pm 10.36$ & 0.0018 & $43.74 \pm 11.98$ & $47.74 \pm 11.78$ & 0.0021 \\
\hline Cytotoxic T lymphocytes (CD8 $8^{+}$) & $26.39 \pm 9.42$ & $23.95 \pm 8.26$ & 0.0001 & $26.48 \pm 7.13$ & $24.08 \pm 8.01$ & 0.0189 \\
\hline$\% \mathrm{CD}_{4}^{+} / \% \mathrm{CD} 8^{+}$ratio & $1.93 \pm 0.97$ & $2.31 \pm 1.20$ & 0.0001 & $1.82 \pm 0.82$ & $2.25 \pm 1.05$ & 0.0001 \\
\hline T reg lymphocytes (CD4+/CD25+) & $2.27 \pm 1.04$ & $2.86 \pm 1.64$ & 0.0006 & $2.34 \pm 1.51$ & $3.03 \pm 1.78$ & 0.0011 \\
\hline
\end{tabular}

SD - standard deviation, NK cells - natural killer cells, $p$ - statistical significance. 
cell level decrease was statistically significant only in the symptomatic subgroup $(19.41 \pm 9.30$ before CEA and $16.52 \pm 9.37$ after CEA; $p=0.0044)$, but not in the asymptomatic subgroup (17.88 \pm 9.14 before CEA and $15.91 \pm 9.51$ after CEA; $p=0.0886$ ), (Figure 1). The percentages of monocytes after CEA in both subgroups were decreased, although this observation did not reach statistical significance. On the other hand, the following IC species showed an increase 6 hours after CEA: B lymphocytes, $T$ helper lymphocytes and Treg lymphocytes $\left(C D 4^{+} / C D 25^{+}\right)$, which was significant in both subgroups. The T lymphocyte level showed a statistically significant increase only in the symptomatic patients $(69.74 \pm 10.16$ before CEA vs. $71.45 \pm 9.77$ after CEA; $p=0.0462)$, but not in the asymptomatic subgroup (70.08 \pm 11.19 prior to CEA and 70.21 $\pm 12.35 ; p=0.9048$ ), (Figure 2). A comparison of $\% \mathrm{CD}^{+} / \% \mathrm{CD}^{+}$ratio before CEA and $6 \mathrm{~h}$ after the procedure showed statistically significant differences in both symptomatic and asymptomatic patients.

\section{Discussion}

In this prospective observational study the percentages of peripheral immune cells, both innate and adaptive immunity, prior to carotid endarterectomy and $6 \mathrm{~h}$ after carotid artery eversion were analyzed in symptomatic and asymptomatic patients. The results indicate that the injury to the carotid arterial wall triggers a significant response in the peripheral immune cell subsets $6 \mathrm{~h}$ after the insult. To the best of our knowledge, this is the first description available in the literature regarding these cell species during carotid artery eversion, which is a major vascular injury to the abnormal, diseased, atherosclerotic vessel wall.

Based on many human and animal studies, atherosclerosis is regarded as a chronic inflammatory disease, initiated and perpetuated by innate and adaptive immunity $[19,20]$. Another definition for atherogenesis is the imbalance between immune cells producing pro-atherogenic mediators and regulatory $T$ cells with immunosuppressive, antiatherogenic properties [21]. It is known that the inflammatory status is higher in the immune cells of the carotid plaque, as compared to those cells in the peripheral blood [9]. Most of the findings available in the literature concentrate on the immune composition of the carotid plaque. The evidence that both innate and adaptive mechanisms play a major role in atherosclerotic plaque progression and instability is clear and widely available. However, data regarding circulating immune cell subsets are very scarce. The hypothesis for our study was that there is a difference between the symptomatic and asymptomatic subgroups re-

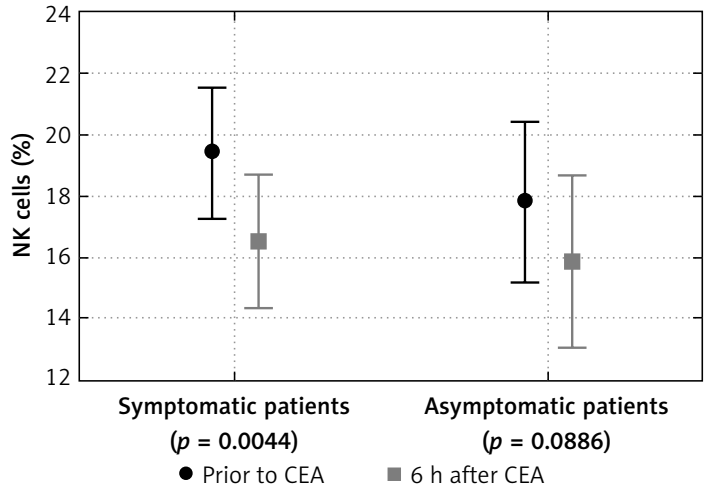

Figure 1. Peripheral NK cells in symptomatic and asymptomatic subgroups prior to and 6 hours after carotid endarterectomy

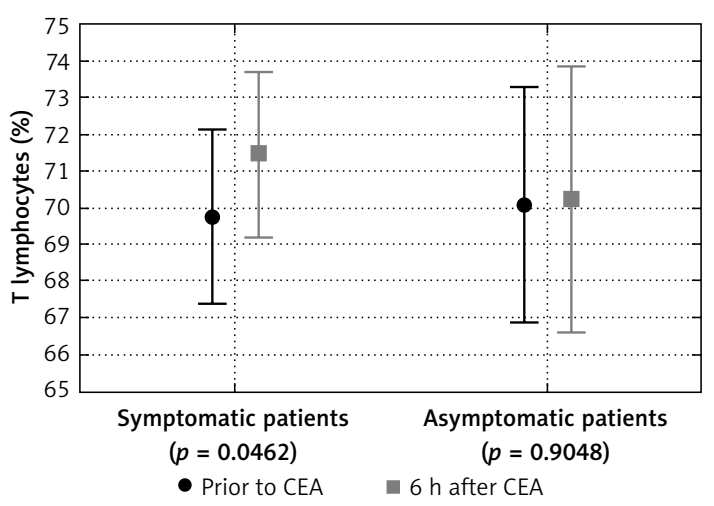

Figure 2. T lymphocytes in symptomatic and asymptomatic subgroups prior to and 6 hours after carotid endarterectomy

garding circulating immune cell percentages that could serve as means of monitoring the carotid plaque instability.

This study shows that some changes in the circulating IC percentages are specific and statistically significant only in the symptomatic patients. This observation includes an NK cell level decrease (19.41 \pm 9.30 before CEA and 16.52 \pm 9.37 after CEA; $p=0.0044)$ and a T lymphocyte level increase $(69.74 \pm 10.16$ before CEA vs. 71.45 \pm 9.77 after CEA; $p=0.0462$ ) in the symptomatic subgroup. On the other hand, our analysis shows some statistically significant changes in both subgroups and this includes an increase in the percentages of B lymphocytes, $T$ helper lymphocytes and Treg lymphocytes (CD4 $\left.{ }^{+} / \mathrm{CD} 25^{+}\right)$.

It has been widely discussed that the immune cells are relevant clinical biomarkers after cerebrovascular episodes (ischemic stroke, TIA, amaurosis fugax). Acute decompensation of the cerebrovascular circulation, presenting as stroke or TIA, is a consequence of carotid atheromatous plaque instability due to an increase in the activity of inflammatory cells [10]. They become a relevant source of pro-inflammatory cytokines including TNF- $\alpha$, IFN- $\gamma$, IL- $1 \beta$ and IL- 6 , which not only perpet- 
uate the inflammatory response after endothelial injury, but also initiate repair mechanisms $[11,17]$. They also serve as potential therapeutic intervention targets; therefore a detailed characterization of their behavior during CEA seems necessary to understand the effect of immunodepression in the early postoperative period. It must be appreciated that many demographic and non-modifiable clinical variables are present, making any prediction as to the influence of the procedure on the immune system very difficult.

Studies concentrating on the identification of $T$ lymphocyte species in the atheromatous plaques of carotid arteries lead to the conclusion that the main effectors are CD4 ${ }^{+}$cells, whereas the role of $\mathrm{CD}^{+}$cells in atherosclerosis has not been fully elucidated. Rohm et al. showed that in unstable lesions, the numbers of pro-inflammatory cells such as T helper cells, cytotoxic T cells, and natural killer cells were significantly higher as compared to stable plaques, whereas the unstable lesions contained significantly lower numbers of Tregs. The authors suggest that the imbalance of local pro- and anti-inflammatory immune cells may increase the inflammatory state in vulnerable atheromatous plaques [21].

The data from available published sources have shown that the majority of T lymphocytes within the atheromatous plaque are CD4+ (T helper) $[22,23]$. Their key role is associated with further differentiation into a pro-atherosclerotic Th1 subgroup and an anti-atherosclerotic Th2 subgroup [24]. In our study the percentage of $\mathrm{T}$ helper lymphocytes increased after carotid endarterectomy in both symptomatic and asymptomatic patients. The results published by Profumo et al. have shown that there is no correlation between the $T$ cell phenotype in peripheral blood and the severity of atherosclerosis in the carotid artery. According to Profumo et al., only cytokines such as TNF- $\alpha$, IFN- $\gamma$ and IL-4 serve as markers of unstable atherosclerosis and the changes in the percentages of circulating lymphocytes do not correlate with advancing atherosclerosis [11].

The role of Tregs (CD4+CD25+) has been described to play a crucial role in pathological Th1 activation against autoantigens, a process controlling $\mathrm{T}$ cell immunological homeostasis [25-27]. It has been shown that a deficit of Treg $\left(\mathrm{CD} 4^{+} \mathrm{CD} 25^{+}\right)$lymphocytes accelerates atherosclerosis in experimental models $[28,29]$. In our study the percentage of Treg lymphocytes $\left(\mathrm{CD} 4{ }^{+} \mathrm{CD} 25^{+}\right)$ increased after CEA in both subgroups. This result may be related to a redistribution of this lymphocyte subpopulation between circulating blood and the carotid vessel. The dynamics of the Treg response after CEA has not been previously described, but it may be associated with Treg activity to suppress the local inflammatory response after surgical insult to the diseased vessel [30].
The innate immunity (NK cells, macrophages, neutrophils) constitutes an important process in atheromatous plaque pathology, which according to Whitman et al. decreases the disease severity $[31,32]$. We found no studies evaluating NK cell levels after carotid endarterectomy. In our study the levels of peripheral (circulating) NK cells were significantly lower in the symptomatic patients as compared to asymptomatic subgroups, but the causal relationship warrants further investigation. According to Clerc et al., patients with severe atherosclerosis present with a significant increase in the NK cell level prior to revascularization [33]. Similarly, Bruunsgaard et al. found that elderly patients with atherosclerosis have a higher concentration of NK cells with a decrease in their cytotoxicity [34].

Monocytes are precursors of macrophages that are crucial players in the process of atherosclerosis. Monocytes consist of subsets which can be differentiated according to their expression of the lipopolysaccharide (LPS) receptor CD14 and the FcIll receptor CD16 [35]. In our study the levels of monocytes before and after CEA showed statistically insignificant changes in both symptomatic and asymptomatic patients prior to and after CEA. It has been recognized however that monocyte subsets are major players in atherosclerosis and their presence also leads to plaque instability and rupture [36-38]. Recently, Grosse et al. performed a small pilot study that revealed that absolute levels of monocyte subset counts in the peripheral venous blood may be regarded as markers of histological signs of carotid plaque vulnerability [39]. They showed that Mon2 counts (monocyte subset) are significantly elevated in patients with high-grade symptomatic and asymptomatic carotid stenosis and concluded that the Mon2 subset carries pro-atherogenic and pro-inflammatory potential.

Also the effect of various medications on the immune system must be acknowledged. The use of aspirin was the same in both subgroups as only patients using prophylactic doses were included in the study. Also the use of statins may affect the inflammatory state. The two subgroups in our study showed statistically insignificant differences in the use of statins. The same must be mentioned regarding local anesthetics used to perform regional anaesthesia.

When analyzing the behavior of immune cells in our study we must understand the importance of the observed changes to produce a clinically meaningful patient-centric outcome measure. The significance of the changes in the percentage of circulating peripheral immune cells is yet to be established. This study should be treated as a preliminary analysis of circulating immune cells that brings useful information to evaluate the im- 
mune status of patients undergoing CEA. Further analysis is necessary to correlate the immunology information with clinical outcome, such as mortality, cerebrovascular accidents or cognitive impairment. It must be underlined that while much of the effort of researchers looking at immune cells has been focused on the actual plaque itself, there are not many studies looking at circulating peripheral immune cells and cognitive outcome [40]. Similarly, the inflammatory state and cognitive outcome have been described in a recent publication [41].

Moreover, there are several limitations to this study that should be considered. Firstly, it should be remembered that the time-frame of $6 \mathrm{~h}$ was arbitrarily chosen by the investigators and may not be ideal in capturing the subtle change in immune cell levels after a surgical insult to the carotid artery. Secondly, the current study describes the magnitude of changes before and after carotid endarterectomy, whereas further prospective studies are required to formally elucidate causality. Thirdly, it is not always possible to eliminate the bias originating from non-vascular origin of immune system stimulation, and thus further studies are needed to better understand the underlying pathophysiology.

In conclusion, this is the first study to compare the circulating immune cell subsets in patients undergoing carotid endarterectomy. There were significant differences in circulating immune cell levels before and $6 \mathrm{~h}$ after CEA in both symptomatic and asymptomatic patients. This study enriches our understanding of immune cell kinetics during carotid endarterectomy and may guide future interventions. Nevertheless, it requires further investigation.

\section{Acknowledgments}

The authors would like to thank Ms. Mai NguyenProkowska (Department of Pathomorphology of the Pomeranian Medical University) for assistance with flow cytometry.

Source of funding: Research granted by the Ministry of Science and Higher Education in Poland, NN 403293336.

\section{Conflict of interest}

The authors declare no conflict of interest.

\section{References}

1. Hansson GK, Hermansson A. The immune system in atherosclerosis. Nat Immunol 2011; 12: 204-12.

2. Falk E. Pathogenesis of atherosclerosis. J Am Coll Cardiol 2006; 47 (8 Suppl): C7-12.

3. Liapis CD, Bell PRF, Mikhailidis D, et al. European Society of Vascular Surgery (ESVS) guidelines. Invasive treat- ment for carotid stenosis: indications, techniques. Eur J Vasc Endovasc Surg 2009; 37 (Suppl. 4): 1-19.

4. Brahmanandam S, Ding E, Conte M, Belkin M, Nguyen LL. Clinical results of carotid artery stenting compared with carotid endarterectomy. J Vasc Surg 2008; 47: 343-9.

5. Levy El, Mocco J, Samuelson RM, Ecker RD, Jahromi BS, Hopkins LN. Optimal treatment of carotid artery disease. J Am Coll Cardiol 2008; 51: 979-85.

6. Ammirati E, Moroni F, Magnoni M, Camici PG. The role of $\mathrm{T}$ and $\mathrm{B}$ cells in human atherosclerosis and atherothrombosis. Clin Exp Immunol 2015; 179: 173-87.

7. Jaipersad AS, Lip GYH, Silverman S, Shantsila E. The role of monocytes in angiogenesis and atherosclerosis. J Am Coll Cardiol 2014; 63: 1-11.

8. Ilhan F, Kalkanli ST. Atherosclerosis and the role of immune cells. World J Clin Cases 2015; 3: 345-52.

9. Sternberg Z, Ghanim H, Gillotti KM, et al. Flow cytometry and gene expression profiling of immune cells of the carotid plaque and peripheral blood. Atherosclerosis 2013; 229: 338-47.

10. Profumo E, Buttari B, Tosti M, et al. Association of intracellular pro- and anti-inflammatory cytokines in peripheral blood with the clinical or ultrasound indications for carotid endarterectomy in patients with carotid atherosclerosis. Clin Exp Immunol 2008; 152: 120-6.

11. Profumo E, Siracusano A, Ortona E, et al. Cytokine expression in circulating $T$ lymphocytes from patients undergoing carotid endarterectomy. I Cardiovasc Surg (Torino) 2003; 44: 237-42.

12. Prass K, Meisel C, Höflich C, et al. Stroke-induced immunodeficiency promotes spontaneous bacterial infections and is mediated by sympathetic activation reversal by poststroke Thelper cell type 1-like immunostimulation. J Exp Med 2003; 198: 725-36.

13. Haeusler KG, Schmidt WU, Foehring F, et al. Immune responses after acute ischemic stroke or myocardial infarction. Int J Cardiol 2012; 155: 372-7.

14. Vogelgesang A, Grunwald U, Langner S, et al. Analysis of lymphocyte subsets in patients with stroke and their influence on infection after stroke. Stroke 2008; 39: 237-41.

15. Urra X, Cervera A, Obach V, Climent N, Planas AM, Chamorro A. Monocytes are major players in the prognosis and risk of infection after acute stroke. Stroke 2009; 40: 1262-8.

16. Hansson G, Jonasson L. The discovery of cellular immunity in the atherosclerotic plaque. Arterioscler Thromb Vasc Biol 2009; 29: 1714-7.

17. Profumo E, Esposito C, Buttari B, et al. Intracellular expression of cytokines in peripheral blood from patients with atherosclerosis before and after carotid endarterectomy. Atherosclerosis 2007; 181: 340-7.

18. Adams HP, Bendixen BH, Kappelle LJ, et al. Classification of subtype of acute ischemic stroke. Definitions for use in a multicenter clinical trial. TOAST. Trial of Org 10172 in Acute Stroke Treatment. Stroke 1993; 24: 35-41.

19. Hansson GK, Libby P, Schonbeck U. Innate and adaptive immunity in the pathogenesis of atherosclerosis. Circ Res 2002; 91: 281-91.

20. VanderLaan P, Reardon C. The unusual suspects: an overview of the minor leukocyte populations in atherosclerosis. J Lipid Res 2005; 46: 829-38.

21. Rohm I, Atiskova Y, Drobnik S, et al. Decreased regulatory $T$ cells in vulnerable atherosclerotic lesions: imbalance between pro- and anti-inflammatory cells in atherosclerosis. Mediators Inflamm 2015; 2015: 364710. 
22. Stemme S, Holm J, Hansson GK. T lymphocytes in human atherosclerotic plaques are memory cells expressing CD45RO and the integrin VLA-1. Arterioscler Thromb 1992; 12: 206-11.

23. Zhou X, Stemme S, Hansson GK. Evidence for a local immune response in atherosclerosis: CD4+ T cells infiltrate lesions of apo E-deficient mice. Am J Pathol 1996; 149: 359-66.

24. Baidya SG, Zeng QT. Helper T cells and atherosclerosis: the cytokine web. Postgrad Med J 2005; 81: 746-52.

25. Piccirillo CA, Shevach EM. Cutting edge: control of CD8+ $\mathrm{T}$ cell activation by $\mathrm{CD} 4+\mathrm{CD} 25+\mathrm{T}$ immunoregulatory cells. J Immunol 2001; 167: 1137-40.

26. Sakaguchi S. Regulatory T cells: key controllers of immunologic self tolerance. Cell 2000; 101: 455-8.

27. Shevach EM. Regulatory T cells in autoimmunity. Annu Rev Immunol 2000; 18: 423-49.

28. Ait-Oufella H, Salomon B, Potteaux S, et al. Natural regulatory $T$ cells control the development of atherosclerosis in mice. Nat Med 2006; 12: 178-80.

29. Mallat Z, Ait-Oufella H, Tedgui A. Regulatory T-cell immunity in atherosclerosis. Trends Cardiovasc Med 2007; 17: 113-8.

30. Caligiuri G, Nicoletti A. Tregs and human atherothrombotic disease. Arterioscler Thromb Vasc Biol 2010; 30: 1679-81.

31. Whitman SC, Rateri DL, Szilvassy SJ. Yokoyama W, Daugherty A. Depletion of natural killer cell function decreases atherosclerosis in low-density lipoprotein receptor null mice. Arterioscler Thromb Vasc Biol 2004, 24: 1049-54

32. Linton MF, Major AS, Fazio S. Proatherogenic role for NK cells revealed. Arterioscler Thromb Vasc Biol 2004; 24: 992-4.

33. Clerc G, Rouz PM. Lymphocyte subsets in severe atherosclerosis before revascularization. Ann Intern Med 1997; 126: 1004-5.

34. Bruunsgaard H, Pedersen AN, Schroll M, Skinhøj P, Pedersen BK. Decreased natural killer cell activity is associated with atherosclerosis in elderly humans. Exp Gerontol 2001; 37: 127-36.

35. Passlick B, Flieger D, Ziegler-Heitbrock HW. Identification and characterization of a novel monocyte subpopulation in human peripheral blood. Blood 1989; 74: 2527-34.

36. Merino A, Buendia P, Martin-Malo A, et al. Senescent CD14+CD16+ monocytes exhibit proinflammatory and proatherosclerotic activity. J Immunol 2011; 186: 1809-15.

37. Kaito M, Araya S, Gondo Y, et al. Relevance of distinct monocyte subsets to clinical course of ischemic stroke patients. PLoS One 2013; 8: e69409.

38. Urra X, Villamor N, Amaro S, et al. Monocyte subtypes predict clinical course and prognosis in human stroke. J Cereb Blood Flow Metab 2009; 29: 994-1002.

39. Grosse GM, Schulz-Schaeffer WJ, Teebken OE, et al. Monocyte subsets and related chemokines in carotid artery stenosis and ischemic stroke. Int J Mol Sci 2016; 17: 433.

40. Mocco J, Wilson DA, Ducruet AF, et al. Elevations in preoperative monocyte count predispose to acute neurocognitive decline after carotid endarterectomy for asymptomatic carotid artery stenosis. Stroke 2006; 37: 240-2.

41. Halazun H, Mergeche J, Mallon K, Connolly E, Heyer E. Neutrophil-lymphocyte ratio as a predictor of cognitive dysfunction in carotid endarterectomy patients. J Vasc Surg 2014; 59: 768-73. 